\title{
EDITORIAL \\ FIAT, flow diverters, and establishing the role of new technology
}

\author{
Peter S. Amenta, MD, Ricky Medel, MD, and Aaron S. Dumont, MD \\ Department of Neurosurgery, Tulane University School of Medicine, New Orleans, Louisiana
}

$\mathrm{D}$ ESPITE significant advances in the microsurgical and endovascular management of aneurysms, there continue to be lesions for which the ideal treatment option remains controversial. This group of aneurysms includes those that arise from the proximal intracranial carotid, many posterior circulation aneurysms, dissecting pseudoaneurysms, and dysplastic wide-necked aneurysms that incorporate the parent vessel. Flow diversion devices have been introduced as a means to address these aneurysms, which would otherwise require advanced skull base techniques, possible bypass and vessel sacrifice, or endovascular management beyond simple coiling. The authors have executed a randomized care trial (Flow Diversion in the Treatment of Intracranial Aneurysm Trial [FIAT]) to examine the safety and efficacy of flow diversion compared with the more traditional means of aneurysm treatment. ${ }^{5}$ The study was halted due to safety concerns as a significantly greater number of patients randomized to flow diversion were dead or dependent at 3 or more months. Efficacy, measured by angiographic obliteration, was also below the expectations put in place in the trial hypothesis. As a result, the authors have provided important data that clinicians must take into consideration when addressing these difficult lesions.

Based on the results of the trial, the authors conclude that flow diversion proved to not be as safe and effective as they originally hypothesized. The authors eloquently discuss the limitations of the study and the significant challenges facing the future study of flow diversion devices. Clearly, additional investigation is needed to better understand the role of flow diversion in the management of cerebral aneurysms. Perhaps this statement is the strongest conclusion that is to be taken from this paper. However, there are significant barriers that will have to be overcome to gain meaningful data in further studies.

First, the grouping of aneurysms under a catchall term such as "aneurysms difficult to treat by traditional methods" is misleading and limits our understanding of what can be accomplished with flow diversion and other methods. The argument needs to be made that the lesions grouped together in this paper, and others like it, are distinct disease processes with varying natural histories, clinical presentations, and established clinical outcomes in the literature. Treatment of a posterior circulation dissecting pseudoaneurysm is much different than treating a cavernous aneurysm or wide-necked dysplastic aneurysm. Additionally, the reasoning behind the application of flow diversion is as diverse as the lesions themselves. Flow diversion is applied to proximal carotid aneurysms due to the advanced microsurgical techniques needed to navigate the complex skull base anatomy and cavernous sinus and the difficulty in obtaining proximal control. In cases of fusiform aneurysms or dissecting pseudoaneurysms, flow diversion offers the possibility of simultaneous parent vessel preservation or reconstruction and aneurysm obliteration. Flow diversion, as was the case in this study, may also be applied to aneurysms that have failed other treatments, which have rendered further traditional means challenging or even impossible. Posterior circulation aneurysms amenable to flow diversion often have components of all of these elements, thereby rendering other treatment options less desirable. As a result, it is difficult to conduct a meaningful study that applies flow diversion to all of these lesions. Importantly, ruptured and unruptured aneurysms were also grouped together in this study, which adds another layer of complexity to analysis of the data. The reliance of flow diversion on dual antiplatelet therapy adds significant risk to its use in the setting of acute intracranial hemorrhage.

Second, lesions not amenable to traditional treatment are relatively rare and the accumulation of significant patient cohorts will be challenging. Third, there is significant variability in clinical practice and between specialists pro- 
viding care. A neurosurgeon trained in microsurgery and intracranial bypass may have a different approach to an aneurysm than a practitioner trained only in endovascular intervention. This contrast greatly affects what lesions are considered suitable or unsuitable for flow diversion and introduces significant bias into a particular study. These problems are neither new nor prohibitive, but are wellknown influences on the introduction of any new intervention or technology.

Flow diversion devices have been FDA approved for use in proximal carotid circulation aneurysms, although their off-label use has been applied to challenging lesions in other locations for which no clear treatment consensus exists. ${ }^{3}$ FIAT demonstrated lower morbidity and mortality rates for flow diverters used in the anterior circulation. These results are consistent with other studies in which higher rates of complications were found when these devices were used to treat posterior circulation lesions. ${ }^{1,4,6}$ The numbers in FIAT are small and there is limited follow-up, but this paper does add weight to the evidence that urges caution in the use of flow diverters in the posterior circulation. However, of note, challenging posterior circulation aneurysms pose difficulty for treatment with all currently available options.

Finally, in interpreting the data from this study, it is important to keep two confounding variables in mind. With the introduction of any new technology there is a user learning curve that must be overcome. Flow diverters have a well-established learning curve and the relative rarity of lesions amenable to flow diversion makes the accumulation of significant experience challenging. ${ }^{2}$ There were no criteria established to permit inclusion of practitioners or centers in the study, yet all operators had performed at least 10 flow diversion cases prior to entry. Although care studies are designed to protect patients from learning curves, it should be remembered that flow diversion is no longer so new that there are a limited number of practitioners employing the devices. A substantial number of centers and individuals have significant experience with the devices, their deployment, and the difficult aneurysms they are used to treat. Granted, the majority of complications that led to poor outcomes occurred remotely and were not related to the procedure. However, we do not know if these could have been avoided with different sizing of the devices, use of more than 1 device, or better placement of the device. These are decisions that are often heavily influenced by accumulated experience over a significant number of cases. Future investigations may benefit from stringent practitioner inclusion criteria.

Lastly, platelet function testing, which has become relatively standard prior to placement of flow diverters, was not a requirement in FIAT. Dual antiplatelet therapy is a critical component of successful flow diversion treatment, with both resistance and over-responsiveness potentially leading to complications. Platelet inhibition is of particular interest in cases of ruptured aneurysms treated with flow diversion, where disastrous hemorrhagic complications may occur. Moving forward, the inclusion of platelet inhibition data in future studies may provide important information regarding the true place for the use flow diversion.
Looking ahead, the next investigations of flow diversion must explore the application of the technology to specific lesions. These studies must account for anatomical location, ruptured or unruptured status, anterior versus posterior circulation, and underlying pathology (i.e., dissection). The fact that the complication rate in the present study, which was heavily influenced by the posterior circulation complications, was higher than expected does not mean that flow diversion is not the right treatment for anterior circulation aneurysms. What the data do tell us is that we have a significant amount of work left to do to establish the lesions best suited for this new technology.

https://thejns.org/doi/abs/10.3171/2016.5.JNS161096

\section{References}

1. Fargen KM, Velat GJ, Lawson MF, Mocco J, Hoh BL: Review of reported complications associated with the Pipeline Embolization Device. World Neurosurg 77:403-404, 2012

2. Jabbour P, Chalouhi N, Tjoumakaris S, Gonzalez LF, Dumont AS, Randazzo C, et al: The Pipeline Embolization Device: learning curve and predictors of complications and aneurysm obliteration. Neurosurgery 73:113-120, 2013

3. Murthy SB, Shah J, Mangat HS, Stieg P: Treatment of intracranial aneurysms with pipeline embolization device: newer applications and technical advances. Curr Treat Options Neurol 18:16, 2016

4. Park MS, Albuquerque FC, Nanaszko M, Sanborn MR, Moon K, Abla AA, et al: Critical assessment of complications associated with use of the Pipeline Embolization Device. J Neurointerv Surg 7:652-659, 2015

5. Raymond J, Gentric JC, Darsaut TE, Iancu D, Chagnon M, Weill A, et al: Flow diversion in the treatment of aneurysms: a randomized care trial and registry. J Neurosurg [epub ahead of print November 4, 2016. DOI: 10.3171/2016.4.JNS152662]

6. Siddiqui AH, Abla AA, Kan P, Dumont TM, Jahshan S, Britz $\mathrm{GW}$, et al: Panacea or problem: flow diverters in the treatment of symptomatic large or giant fusiform vertebrobasilar aneurysms. J Neurosurg 116:1258-1266, 2012

\section{Disclosures}

The authors report no conflict of interest.

\section{Response}

\section{Jean Raymond, MD, ${ }^{1}$ Jean-Christophe Gentric, MD, ${ }^{1,2}$ Robert Fahed, MD, ${ }^{1}$ and Tim E. Darsaut, MD ${ }^{3}$}

'Department of Radiology, Centre Hospitalier de l'Université de Montréal, Notre-Dame Hospital, Montreal, Quebec, Canada; ${ }^{2}$ Groupe d'Étude de la Thrombose en Bretagne Occidentale, Brest, France; and ${ }^{3}$ Department of Radiology, Ottawa Hospital, Civic Campus, Ottawa, Ontario, Canada

We thank Amenta et al. for their thoughtful editorial and welcome the opportunity to clarify what we are trying to accomplish with care trials such as FIAT. First, their comment that "the authors have provided important data that clinicians must take into consideration when addressing these difficult lesions" is perhaps overly generous, considering the data we were able to provide contrasted with what everyone would like to have: definite answers regarding how to best manage various difficult aneurysms. Unfortunately, this ideal goal may be beyond our 
reach for the time being, but the comment misses the primary goal of the enterprise, which is to provide clinicians with the means to prudently offer some patients a novel and promising treatment option, but one that has yet to be shown beneficial as compared with alternative management options. It seemed only natural to start with patients for whom past results of more conventional treatments are believed to be unsatisfactory or suboptimal. With this perspective, obtaining reliable data is important, but can only come later, after the recruitment of a sufficient number of participating centers and patients. The crucial and immediate problem is how to ethically offer a novel therapeutic approach, in other words how to experiment with a new procedure, given the present lack of knowledge as to what to do for any "kind of aneurysms." The fact that we will do this in numerous patients (eventually numerous enough to have reliable answers) will be discussed shortly.

We must remember that by using flow diversion, we are both trying novel devices and experimenting with a new therapeutic strategy (reconstructing the parent vessel instead of clipping or coiling the aneurysm). In doing so, we need a declared clinical research context, because we are entering unknown territory, putting patients at risk of unexpected complications as compared with the alternatives we have been using for decades (exemplified in the case of flow diversion by the rupture of unruptured aneurysms or by parenchymal hematomas distant from the aneurysm). In other words, the care trial context is above all, and contrary to common misconceptions, a case-by-case approach. The ethics of caring for patients is given primacy and priority over a desire for new knowledge, and this drives the design choices of this and all care trials. ${ }^{3}$ Instead of placing patients into situations where they might be willing to forego receiving the standard management option, or tempted to accept the new and promising treatment as if it were already standard care, in a care trial the innovative treatment is offered as a $50 \%$ chance of getting the innovation, combined with a $50 \%$ chance of getting the conventional option that would have been offered prior to when the innovation existed. This strategy balances risks (until the promising treatment is actually shown better when practiced in the real world), and reminds both the patient and the clinician that the innovation is not yet to be prescribed as standard care. First the ethics of care, one patient at a time, then eventually the knowledge, when and if available, when the data are eventually analyzed, after having repeated the process in a sufficiently large number of diverse patients.

This last concept, regarding the protective role of the trial in balancing the risks involved in proposing the innovative treatment to current patients, is not universally accepted within our community. Because many colleagues would rather choose a promising option with unknown risks than use a standard management option with known risks, at least in certain patients, we included a registry within the trial, hoping to capture the outcome of flow diversion in all patients. Although we do not recommend this strategy-because by allowing the use of the innovative treatment just as if it was standard care, it fails to protect current patients-flow diversion was already hypothesized to be "so much better" in certain cases by some clinicians that we reasoned that close monitoring of the clinical usage of the innovation would be a way to at least protect future patients if adverse events were alarmingly frequent. Now notice what happened: complications occurred at a worrying frequency, and while no one will ever know what might have happened had alternative options been used for the registry patients, we can at least be somewhat reassured by the valid comparison (although yet poorly powered) of patients offered randomized allocation. No one did significantly better or worse, for the time being, and the independent Data Safety and Monitoring Committee could tentatively judge that the frequency of complications was likely caused by the "kinds of patients" included in the trial, rather than by the dangers inherent in using flow diversion.

This brings us to the second comment ("the strongest conclusion that is to be taken from this paper" is that "additional investigation is needed to better understand the role of flow diversion in the management of cerebral aneurysms"), which is right on target, but the editorial suggestions that follow "there are significant barriers that will have to be overcome to gain meaningful data in further studies" rehearse time-honored misconceptions that, in our view, need to be revised if we are to one day provide outcome-based neurovascular care.

The first suggestion, the most prevalent misconception in our field, concerns heterogeneity. Authors of the editorial appear to believe that meaningful clinical studies can only be about some theoretical truth, such as the "efficacy of flow diversion" in well-defined species of aneurysms, because "the lesions grouped together in this paper...are distinct disease processes with varying natural histories, clinical presentations, and established clinical outcomes in the literature." So what? An elderly patient with heart disease, presenting with aphasia at 5 hours, right hemiplegia, some infarcted territory, and a National Institutes of Health Stroke Scale (NIHSS) score of 28 has a natural history, clinical presentation, and established clinical outcome varying from those of a 40-year-old patient with a traumatic carotid dissection, a good circle of Willis, presenting at 2 hours with a left hemiparesis, a normal CT scan, and an NIHSS score of 8 . When a center is experimenting with thrombectomy, we see no reason why these 2 patients could not be offered participation in the same thrombectomy trial. The study is trialing thrombectomy, not patient age, health status, thrombus etiology, location, severity of deficits, presence of infarction, etc.

Of course, each and every patient is unique, but we can learn from experience. A trial aggregates patients with similarities and differences. We tend to make a priori distinctions between "kinds of patients," kinds that have been identified and then entrenched in our common language and clinical practice because they have been meaningful in some other contexts in the past (surgery of posterior circulation aneurysms, for example). However, for the time being, no one knows whether such distinctions should impact whether the patient should be treated with flow diversion or not. What FIAT patients have in common is "an aneurysm for which flow diversion is considered a promising treatment," a somewhat different concept from the grouping of aneurysms under a catchall term such as "aneurysms difficult to treat by traditional methods." The 
key inclusion criterion is that the clinician considers using flow diversion for the patient. This is because the burden of proof is on flow diversion. Patients can later be distinguished according to any number of aneurysm or patient characteristics, as well as concepts such as "dissections" or "dysplasia" if need be, provided the patients have been included in the trial. In a care trial, the intervention is regulated on a case-by-case basis. The many careful distinctions that we may wish to make may later be shown to be pertinent and meaningful after the conduct of the study (provided we are careful with subgroup analyses), ${ }^{4}$ according to results. Otherwise, in the meantime, selection only serves to deny some patients access to the innovation, or encourage the usage of the innovative treatment outside the prudent context of the trial.

Verifying that treatment works for a large number of clinically diverse patient categories is meaningful. Contrary to what editorialists seem to think, heterogeneity is not a weakness, but a strength. One way to think about this is to contrast theory and practice. What we need right now are care trials, conceived as a science of the ethical practice of an innovative treatment, as performed in the real world of patients for whom a certain treatment appears promising, as opposed to studies looking for causal mechanisms involved in treating a specific disease process with a specific device. Who wants a treatment that is effective in theory, but shown to be harmful in practice? We can be more confident that thrombectomy should be adopted as a standard care if it can be shown to improve outcomes for a wide variety of patients, with distinct presentations and prognoses, when performed by interventionists of varying proficiency, in various circumstances, than when it is shown beneficial only in a narrow subset of selected patients when performed by highly skilled operators. This is the crux of the contrast between explanatory and pragmatic trials that we have reviewed elsewhere. ${ }^{2}$

It is unclear what the authors wish to do with heterogeneity. Multiply an increasing number of trials with narrow selection criteria of an already uncommon condition? Perform an explanatory trial on a small selection of patients, treated by experts working under optimal conditions, and then apply flow diversion to the wide variety of patients treated under real-world suboptimal conditions? We know from the past what the argument about heterogeneity can do, which is lead to no trials at all, and working in the dark forever. To recommend the use of innovative care without randomized evidence is actually more risky than working in the dark. Information can not only be lacking, but worse, some pieces of knowledge can be misleading (Fig. 1). In the absence of the appropriate comparison (the same patients randomized to alternative options), we are left with making treatment decisions based on invalid infer-
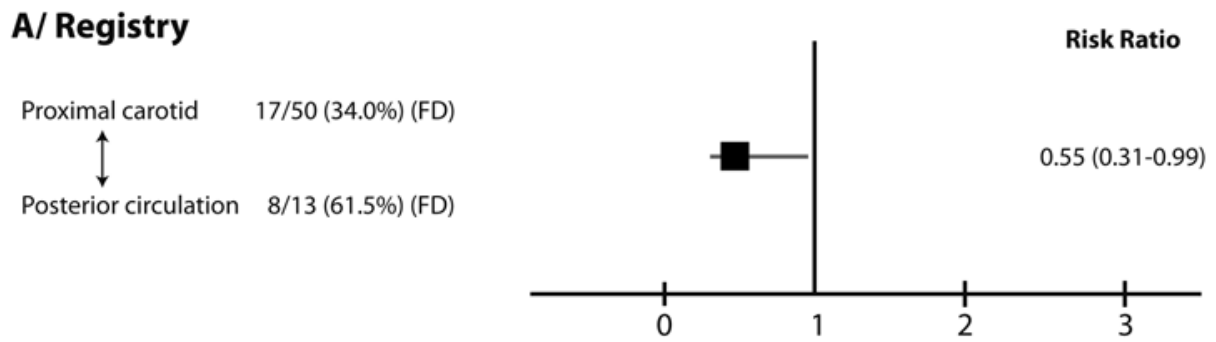

\section{B/ RCT}

Proximal carotid Proximal carotid

$11 / 26(42.3 \%)$ (FD) $10 / 28(35.7 \%)(B S T)$

Posterior circulation Posterior circulation

$4 / 7(57.1 \%)($ FD) $3 / 7(42.9 \%)(B S T)$

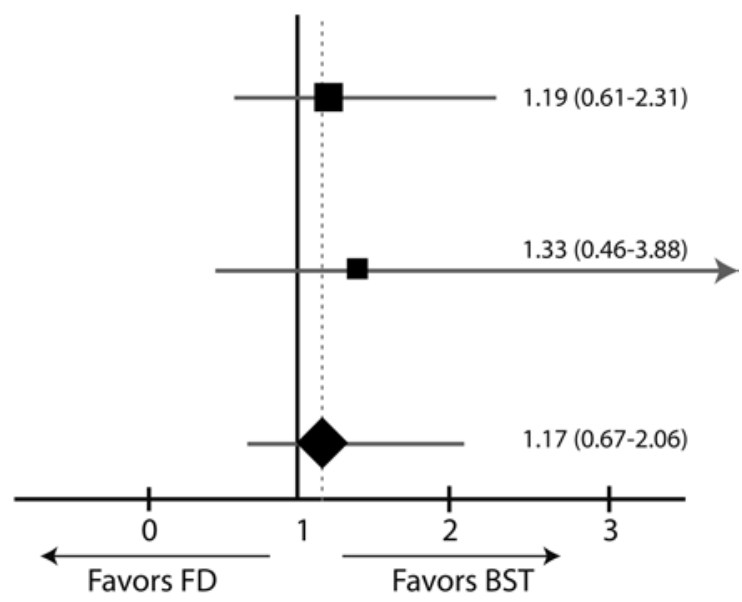

FIG. 1. Dangerous inferences from invalid comparisons versus valid randomized comparisons. Panel A presents the relative risk (and 95\% confidence interval) of poor outcome from flow diversion (FD), when in the absence of a randomized controlled group, proximal carotid and posterior circulation aneurysms are compared (actual data from FIAT: all patients who received flow diversion). Observers are tempted to erroneously conclude that flow diversion is indicated for proximal carotid aneurysms, but cautioned against its use in posterior circulation aneurysms. Panel B is a forest plot of the relative risk (and $95 \%$ confidence interval) of poor outcome following flow diversion versus best standard treatment (BST), for all patients randomized in FIAT, as well as for the proximal carotid and posterior circulation subgroups. Flow diversion has yet to be shown superior to BST for any kind of patient, including patients with proximal carotid aneurysms. RCT = randomized controlled trial. 
ences from inappropriate comparisons, such as comparing anterior and posterior circulation aneurysms. ${ }^{1}$ No matter how true the fact that posterior circulation aneurysms may do worse than anterior circulation aneurysms, and no matter how much this may affect absolute numbers regarding overall trial results, this truth has no bearing on the decision as to whether a patient with an anterior or posterior circulation aneurysm should be treated with flow diversion or with a more conventional alternative.

Returning to the problem of similarities and differences when trying to learn from experience, the important thing to do is not to restrict access to the trial, and group together only patients who are similar in some or another respect (but always different regarding many other aspects, depending on how far you care to look). The important thing to do is to validly compare 2 groups that similarly aggregate all sorts of patients that differ in all kinds of aspects, but that in the end (with a sufficiently large number of patients) are similar in all respects, except for the one variable we wish to study: having received the intervention under investigation or not, in this particular case, flow diversion.

Admittedly, such pragmatic trials may be more complex to analyze when the time comes to draw conclusions, as the editorialists remind us. That is the inevitable consequence of trying to ethically manage patients on a caseby-case basis, and then looking for reliable knowledge regarding real world practices, as opposed to imposing experiments on patients that aim for theoretical truths that may not apply in practice.

In spite of what may have been perceived as an alarming frequency of complications, we still believe flow diversion to be a promising treatment, at least for some patients. The problem is that it has yet to be shown beneficial for any kind of patient. For this reason, the FIAT trial continues to enroll patients for whom flow diversion appears promising. Whether reliable answers will be obtained depends on the recruitment of a sufficient number of centers and collaborators. In the meantime, we believe the way to practice innovative care in the best interest of current patients is within the context of a care trial.

\section{References}

1. Brinjikji W, Murad MH, Lanzino G, Cloft HJ, Kallmes DF: Endovascular treatment of intracranial aneurysms with flow diverters: a meta-analysis. Stroke 44:442-447, 2013

2. Magro E, Gentric JC, Darsaut TE, Ziegler D, Bojanowski MW, Raymond J: Responses to ARUBA: a systematic review and critical analysis for the design of future arteriovenous malformation trials. J Neurosurg [epub ahead of print April 29, 2016. DOI: 10.3171/2015.6.JNS15.619]

3. Raymond J, Darsaut TE, Altman DG: Pragmatic trials can be designed as optimal medical care: principles and methods of care trials. J Clin Epidemiol 67:1150-1156, 2014

4. Rothwell PM: Treating individuals 2. Subgroup analysis in randomised controlled trials: importance, indications, and interpretation. Lancet 365:176-186, 2005 